\title{
Tactual and visual learning of forms differing in degree of symmetry'
}

\author{
Richard D. Walk \\ GEORGE WASHINGTON UNIVERSITY
}

\begin{abstract}
One group learned to associate nonsense syllables with forms by sight, and then was tested on cross-modal transfer to the same forms using touch. The other group had the opposite procedure. The visual group performed best in learning the symmetrical forms while the tactual group's best performance was on the less symmetrical forms. Cross-modal transfer of the groups was similar.

\section{Problem}

A person blinded from birth learns to identify objects by touch. If this individual obtains sight, as in an operation to remove congenital cataracts, objects formerly recognized by touch eventually are recognized by vision. In its simplest form, the recognition of objects by sight after they have been learned by touch is simply a problem in transfer of trianing, or, as it is called in this context, cross-modal transfer. In studying recognition of objects by sight after tactual learning, there are actually two problems. The first, mentioned previously, is the problem of transfer, information received through one sense modality is compared to information given through another. The second problem concerns the acquisition of information through the sense modalities themselves: do the same "principles" govern learning by touch that govern learning by vision?

In the case of those born blind who are given sight the two problems intermingle since, if something "different" is learned through touch as compared to that which is learned through vision, then this might affect the cross-modal transfer. Hebb (1949) reviewed the cataract cases cited by von Senden (1932) and concluded that forms recognized instantly by sight (like triangle, circle) were the result of a long visual learning process. Patients born blind had difficulty in learning to recognize forms although some other types of visual recognition were rapid (color, figure-ground perception).

The present experiment was carried out to determine similarities and differences in learning to associate names with forms using the tactual and visual modalities. Both the acquisition of learning and cross-modal transfer were studied.

\section{Method}

Ss, college students, were required to learn to associate nine forms with nine different nonsense syllables. The tactual group, blindfolded, learned to associate by touch the syllables with flat wooden blocks cut out to yield the forms. Following this, they transferred to visual learning: the same forms were used but they were two dimensional rather than three dimensional (black forms on a white background). The visual group had the reverse procedure: Ss learned to associate the two dimensional black figures with nonsense syllables and then were blindfolded to test for transfer tactually. Five sec. were allowed on each form for each group. Ten Ss were in each group.

To iron out unsought idiosyncracies in learning, four different pairings of nonsense syllables and forms were prepared. For any given order a tactual learner was paired with a visual learner.

The forms themselves consisted of two types: three were symmetrical on two axes (double symmetry) and six were symmetrical on one axis (single symmetry). For simplicity these figures will be referred to as the symmetrical figures (double symmetry) and non-symmetrical figures (single symmetry).

\section{Results}

The visual group learned to associate the forms with the appropriate nonsense syllables in an average of 9.7 trials (s.d., 5.4 trials) and the tactual group in an average of 13.0 trials (s.d., 6.3 trials). The difference was not significant, nor was such a comparison a major purpose of this study. In cross-modal transfer the two groups were similar: an average of 1.8 errors on the transfer trial or $80 \%$ cross-modal transfer whether the transfer was from touch to vision or vision to touch, a transfer in agreement with that usually found in studies of this type (Gaydos, 1956).

Differences between groups appeared, however, in learning the two types of forms. As Fig. 1 shows the two groups learned the nonsymmetrical forms at about the same rate, but the symmetrical forms were learned more rapidly by the visual group. In an analysis of variance the interaction of modality with type of figure was highly significant $\left(F_{1,18}=22.42 ; p<.01\right)$. The visual group learned the symmetrical figures more quickly than the tactual group learned them $\left(F_{1,36}=7.04 ; p<.02\right)$, but there were no differences between groups in learning the nonsymmetrical figures $\left(F_{1,36}=0.26\right.$; n.s.). The visual group learned the symmetrical figures more easily than it learned the nonsymmetrical ones $\left(F_{1,18}=\right.$ 16.91; $p<.01$ ) while the tactual group learned the nonsymmetrical figures more easily than it learned the symmetrical forms $\left(\mathrm{F}_{1,18}=6.68 ; \mathrm{p}<.05\right)$.

In the first cross-modal transfer trial the visual group identified $93 \%$ of the symmetrical figures and $74 \%$ of the nonsymmetrical figures by touch. The tactual group, in the cross-modal transfer trial, correctly identified, using vision, $87 \%$ of the symmetrical figures and $77 \%$ of 

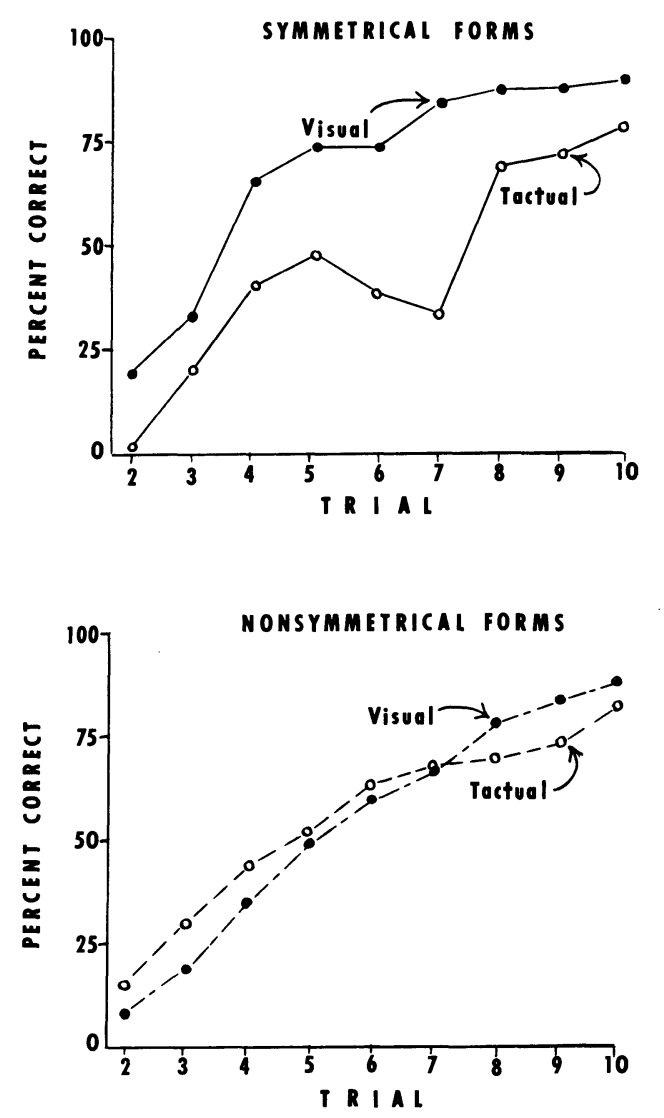

Fig. 1. The acquisition learning curves for the visual learning group as compared to the tactual learning group for symmetrical forms and for nonsymmetrical forms.

the nonsymmetrical ones. The trend was for both groups to identify the symmetrical figures better than the nonsymmetrical ones in cross-modal transfer, but the over-all comparison was not quite significant $(t=2.06$ where 2.10 is needed for $p<.05)$. Cross-modal transfer of both groups, thus, seemed similar.

\section{Diseussion}

The main importance of this experiment is not in cross-modal transfer, which was similar for both groups, but that it shows some qualities of form may be learned more easily by the visual modality than they are learned by the touch modality. The superiority of the visual modality was in the aspects of form implicitly specified by Hebb, redundant visual stimulation where a part may serve for the whole, as in a triangle, circle or square. (The present study has been replicated, with different forms by Milne, 1963.)

Whether the superiority of vision in learning symmetrical figures is representative of the modality qua modality or whether the economy of visual coding is learned, as Hebb suggests, cannot be determined from these data. If young sighted children learn symmetrical and nonsymmetrical figures at the same rate, or, if blind adults use the touch modality to learn symmetrical figures before nonsymmetrical ones, a learning explanation would be favored. But, if young sighted children behave like sighted adults, and, if blind adults learn symmetrical figures at about the same rate as the nonsymmetrical ones, then the present results would be indicative of modality differences in the learning of forms with touch and with vision. Revesz (1950) evidently favored the latter explanation. He claimed that the gestalt laws of perception were restricted in generality and maintained that these laws were laws of visual perception, not laws of tactual or haptic perception.

\section{References}

GAYDOS, H. F. Intersensory transfer in the discrimination of form. Amer. J. Psychol., 1956, 69, 107-110.

HEBB, D. O. The organization of behavior. New York: Wiley, 1949. MILNE, A. M. Visual versus tactual form learning. Paper presented at meetings of the Eastern Psychological Association, 1963. REVESZ, G. Psychology and art of the blind. London: Longmans, Green, 1950 .

SENDEN, M. v. Raum- und Gestaltauffasung bei operierten Blindgeborenen vor und nach der Operation. Leipzig: Barth, 1932. Translated by P. Heath as Space and sight. Glencoe, Ill.: Free press, 1960.

\section{Note}

1. I thank Jesse Smith for his assistance in the conduction of this experiment. Analysis of the results was aided by a grant from the National Institutes of Health (MH-06967-01). 